\title{
On the maximum angle condition for the conforming longest-edge $n$-section algorithm for large values of $n$
}

\author{
Sergey Korotov ${ }^{1,2}$, Ángel Plaza ${ }^{3}$, José P. Suárez ${ }^{3}$
}

November 18, 2013

\author{
${ }^{1}$ BCAM - Basque Center for Applied Mathematics \\ Alameda de Mazarredo 14, E-48009 Bilbao \\ Basque Country, Spain \\ e-mail: korotov@bcamath.org \\ ${ }^{2}$ IKERBASQUE, Basque Foundation for Science \\ E-48011, Bilbao, Spain \\ ${ }^{3}$ Division of Mathematics, Graphics and Computation (MAGiC) \\ IUMA, Information and Communication Systems \\ University of Las Palmas de Gran Canaria \\ Canary Islands, Spain \\ e-mail: \{aplaza@dmat.ulpgc.es\} \{josepablo.suarez@ulpgc.es\}
}

\begin{abstract}
In this note we introduce the conforming longest-edge $n$-section algorithm and show that for $n \geq 4$ it produces a family of triangulations which does not satisfy the maximum angle condition.
\end{abstract}

Keywords: conforming longest-edge $n$-section, minimum angle condition, maximum angle condition, finite element method, mesh refinement

\section{Mathematics Subject Classification: 65N50, 65N30}

\section{Introduction}

The classical longest-edge (LE-) bisection algorithm bisects simultaneously all triangles by medians to the longest edge of each triangle in a given triangulation. In this way, an infinite sequence of nested triangulations can be generated. However, this type of refinements may lead, in general, to the so-called hanging nodes and thus refined triangulations may not all be conforming, in general (see Figure 1). However, many real-life applications where triangulations are used, e.g. the calculations by the finite element method (FEM), require the property of conformity [3]. Therefore, in [5] a modified version of the classical LE-bisection was introduced, where only elements sharing the longest edge of the whole triangulation are bisected at each step (see Figure 2). In this way, all produced triangulations are conforming a priori and therefore this algorithm is called the conforming LE-bisection algorithm. The same idea can, obviously, be used for simplicial meshes in any dimension. 

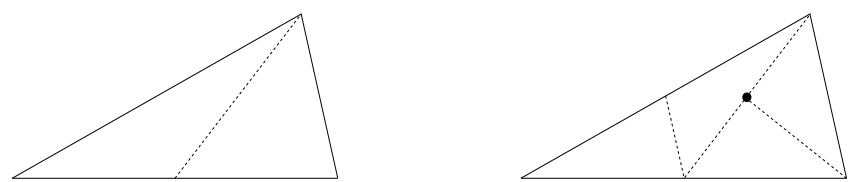

Figure 1: The classical LE-bisection algorithm produces, in general, nonconforming triangulations.
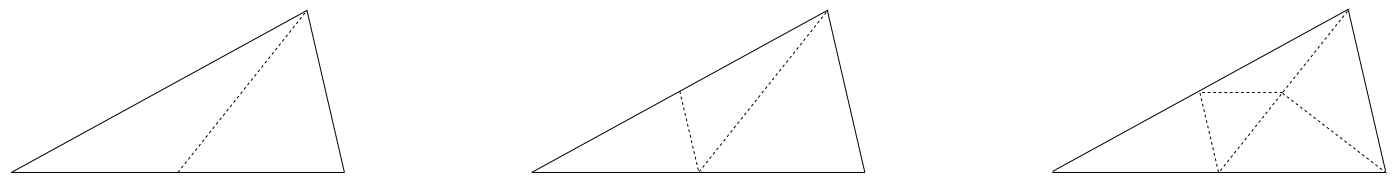

Figure 2: A modified LE-bisection algorithm that always produces conforming triangulations.

In [8], the classical LE-bisection algorithm was generalized in another direction. It was proposed to divide the longest edges into $n$ equal parts (with $n \geq 2$ ), one calls this technique the $L E n$-section. The performance of this algorithm was analysed in [7] and [8] for different values of $n$ when $n \geq 3$. However, as in the classical bisection-version, the LE $n$-section algorithm may produce hanging nodes.

In this work, we blend the ideas of [5] and [8] and define the conforming LE n-section algorithm as follows:

a) In the given triangulation we select the longest edge;

b) For two (or one, if the longest edge lies on the boundary) triangles adjacent to this longest edge we apply the LE $n$-section from [8], and thus we generate a new triangulation. If necessary, we go to the step a).

It is clear that we avoid producing hanging nodes by the above defined algorithm in principle. Obviously, just the same idea can be applied to simplicial meshes in any dimension.

\section{Main results}

Let $\Omega$ be a bounded polygonal domain with a boundary boundary $\partial \Omega$. In what follows we only deal with conforming triangulations of $\bar{\Omega}:=\Omega \cup \partial \Omega$, i.e. an intersection of any two triangles in any triangulation considered is empty, a node, or their adjacent edge. Any triangulation will be denoted by the symbol $\mathcal{T}_{h}$, where $h$ is the so-called discretization parameter, equal to the length of the longest edge in $\mathcal{T}_{h}$.

Definition 1. The (infinite) sequence of triangulations $\mathcal{F}=\left\{\mathcal{T}_{h}\right\}_{h \rightarrow 0}$ of $\bar{\Omega}$ is called a family of triangulations if for every $\varepsilon>0$ there exists $\mathcal{T}_{h} \in \mathcal{F}$ with $h<\varepsilon$.

In $[9,10]$ the following minimum angle condition was introduced: there should exist a constant $\alpha_{0}$ such that for any triangulation $\mathcal{T}_{h} \in \mathcal{F}$ and any triangle $K \in \mathcal{T}_{h}$ we have

$$
0<\alpha_{0} \leq \alpha_{K},
$$

where $\alpha_{K}$ is the minimal angle of $K$. Under this condition various a priori error estimates for the finite element method (FEM) applied to some elliptic problems are usually derived [3]. 
Later condition (1) was weakened in [1, 2, 4], (see also [6]) and the so-called maximum angle condition was proposed: There exists a constant $\gamma_{0}$ such that for any triangulation $\mathcal{T}_{h} \in \mathcal{F}$ and any triangle $K \in \mathcal{T}_{h}$ we have

$$
\gamma_{K} \leq \gamma_{0}<\pi
$$

where $\gamma_{K}$ is the maximum angle of $K$.

Remark 1. Condition (1) obviously implies (2), since $\gamma_{K} \leq \pi-2 \alpha_{K} \leq \pi-2 \alpha_{0}=: \gamma_{0}$, but the converse implication does not hold.

In what follows we will prove that for $n \geq 4$ the conforming LE $n$-section produces triangulations which do not satisfy the maximum angle condition, i.e. there is an infinite sequence of angles in some triangles, among those appearing during the refinement process, which tends to $\pi$ as the LE $n$-section proceeds.

Lemma 1. Let us $n$-sect the triangle with edges of the length $a, b$, and $c$, where $a \leq b \leq c$. Then there exists a positive constant $\kappa=\kappa(n)<1$ such that the lengths of all newly generated sub-edges are not greater than $\kappa c$.

Proof: First, we notice that all the newly generated sub-edges obtained by $n$-secting the edge $c$ are of the length $\frac{1}{n} c$. Further, using the Cosine theorem, we can easily show that the longest generated interior edge is not greater than $\frac{\sqrt{n^{2}-n+1}}{n} c$. Therefore, we can take $\kappa:=\frac{\sqrt{n^{2}-n+1}}{n}<1$.

Theorem 1. The conforming LE n-section algorithm produces the family of triangulations.

Proof: Let $T$ be a triangle which we want to $n$-sect. All $2 n-1$ newly generated edges will be shorter than the longest edge of $T$ (cf. Lemma 1). Therefore the length of the longest edge in the whole triangulation makes a nonincreasing sequence. Its limit exists, and we show now that it is equal to zero.

Let $\varepsilon$ be an arbitrary positive number. Let $e^{\star}$ be the longest edge (if we have several edges of the same length - then it is that one which we $n$-sect first) in the initial triangulation and let $\left|e^{\star}\right| \geq \varepsilon$. After we $n$-sect the edge $e^{\star}$, we get at most $3 n-2$ new edges, whose lengths are not greater than $\kappa\left|e^{\star}\right|$. Further, let $N$ denote the number of edges in the initial triangulation whose lengths are not less than $\varepsilon$, and let $q$ be the number such that $\kappa^{q}<\frac{\varepsilon}{\left|e^{*}\right|}$. Then we observe that at most after $N(3 n-2)^{q-1} n$-section steps all lengths in the resulting triangulation will be less than $\varepsilon$.

Theorem 2. Let $n \geq 4$. The iterative application of the classical LE $n$-section algorithm from [7, 8] to a given arbitrary triangle generates an infinite sequence of subtriangles whose maximum angles tend to $\pi$.

Proof: Let $t$ be the given triangle. Without a loss of generality, let its longest edge be equal to 1 and its angles $\alpha, \beta$, and $\gamma$ be such that $\alpha \leq \beta \leq \gamma$. We may assume that $t$ has two vertices at the points $(0,0)$ and $(1,0)$, with the minimum angle $\alpha$ at the vertex $(1,0)$, and the maximum angle $\gamma$ at the vertex $z$ opposite to its longest edge (see Figure 3). Notice that in this situation, considering $z$ as a complex number, the point $z$ lies in the 
subset of the complex plane $\{z$ with $|z-1| \leq 1, \operatorname{Re}(z) \leq 1 / 2$, and $\operatorname{Im}(z)>0\}$ marked in Figure 3 (left). The LE $n$-section is applied to triangle $t$ resulting in $n$ new subtriangles (see Figure 3 (a) for $n=4$ ).

In this subdivision the triangle having the smallest angle, denoted by $\xi$, is the righthand side triangle with the angle $\alpha$, see Figure 3 (a).

As the longest-edge is divided into $n$ equal parts and $n \geq 4$, we have $\left|z-\frac{n-1}{n}\right|>\frac{1}{n}$ and, therefore, $\xi<\alpha$ (we note that both $\xi$ and $\alpha$ are acute). We will now represent $\xi$ as a function of $n, \beta$, and $\alpha$. From the analysis of values of $x, y$ and $h$ from Figure 3 (b), the following system of linear equations appears

$$
\left.\begin{array}{rl}
y \tan \beta-h & =0 \\
x \tan \alpha-h & =-\frac{1}{n} \tan \alpha \\
x+y & =\frac{n-1}{n}
\end{array}\right\} .
$$

Its solution is

$$
h=\frac{\tan \alpha \cdot \tan \beta}{\tan \alpha+\tan \beta}, \quad y=\frac{\tan \alpha}{\tan \alpha+\tan \beta}, \quad x=\frac{\tan \beta}{\tan \alpha+\tan \beta}-\frac{1}{n} .
$$

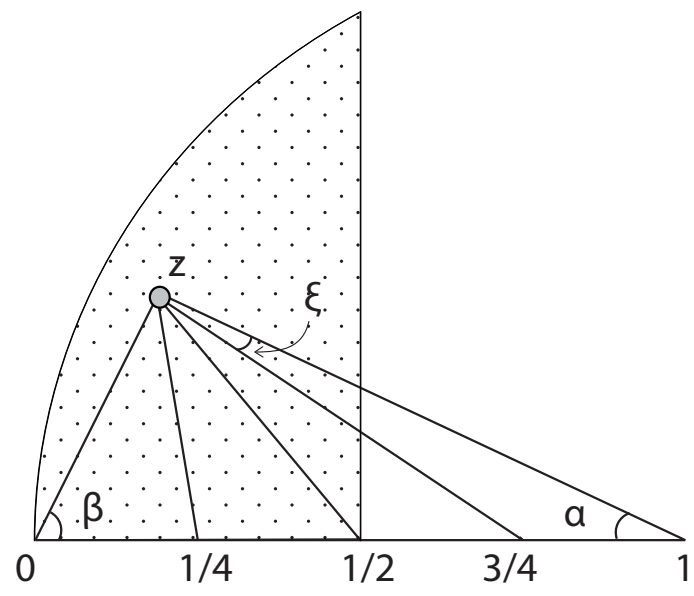

(a)

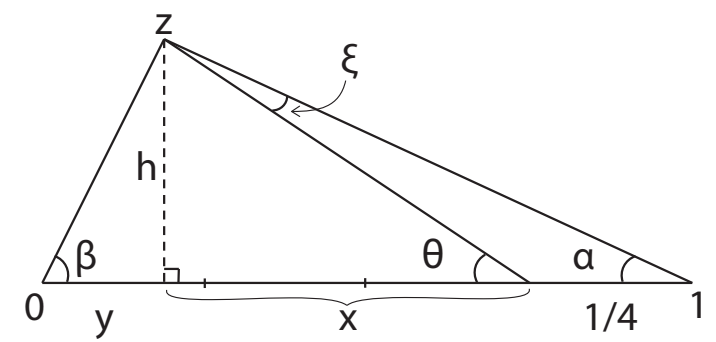

(b)

Figure 3: A triangle sample and denotations for angles for the LE $n$-section with $n=4$.

Note that $\xi=\theta-\alpha$, where $\tan \theta=\frac{h}{x}$, and then

$$
\xi=(\alpha, \beta, h)=\arctan \left[\frac{n \tan \alpha \cdot \tan \beta}{(n-1) \tan \beta-\tan \alpha}\right]-\alpha .
$$

Since $\frac{\partial \xi}{\partial \alpha}>0$ and $\frac{\partial \xi}{\partial \beta}<0, \xi=\xi(\alpha, \beta, h)$ is increasing in $\alpha$ and decreasing in $\beta$.

As $\alpha \leq \beta \leq \frac{\pi-\alpha}{2}$ then $\xi(\alpha, \beta, n) \leq \xi(\alpha, \alpha, n)=\arctan \left(\frac{n}{n-2} \tan \alpha\right)-\alpha$.

For the sake of clarity we will denote by $\xi_{0}$ the maximum possible value of $\xi$ for a given minimum angle $\alpha$. I.e. $\xi_{0}=f(\alpha)=\arctan \left(\frac{n}{n-2} \tan \alpha\right)-\alpha$.

From now on we denote by $(\alpha, \beta)$ a triangle in which its angles are $\alpha \leq \beta \leq \gamma$. We consider the following sequence of triangles: $t_{0}=(\alpha, \beta), t_{1}=\left(\xi_{0}, \alpha\right)=(f(\alpha), \alpha)$, 
$t_{2}=(f(f(\alpha)), f(\alpha))$ and in general $t_{k}=\left(f^{k}(\alpha), f^{k-1}(\alpha)\right)$. Note that by construction, each triangle $t_{k}$ for $k \geq 1$ is related to the corresponding right-hand triangle in the LE $n$-section of a previous triangle in the sense that the minimum angle is greater than the minimum angle of the right-hand triangle, and the second minimum angle is equal to the corresponding second minimum angle.

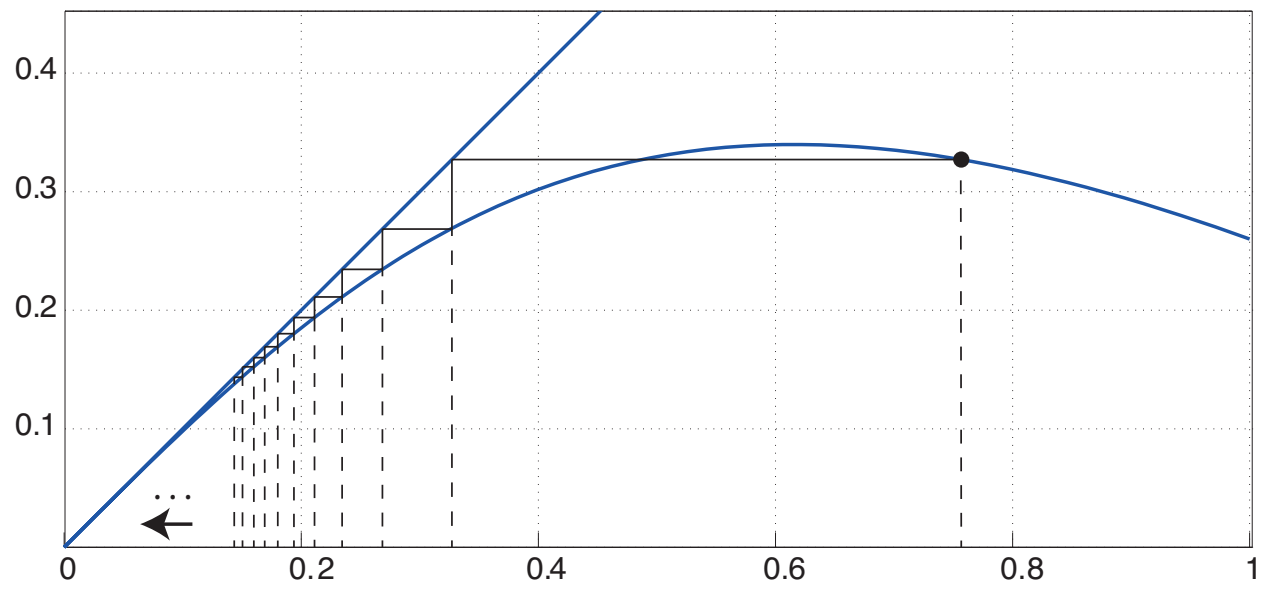

Figure 4: Graphed curves $\alpha$ and $\alpha_{k}=f\left(\alpha_{k-1}\right)=\arctan \left(2 \tan \left(\alpha_{k-1}\right)\right)-\alpha_{k-1}$.

Since $f^{\prime}(x)=\frac{2}{n-2}\left|\frac{1-\frac{n}{n-2} \tan ^{2} x}{1+\left(\frac{n}{n-2}\right)^{2} \tan ^{2} x}\right|<1$ for $x \in\left(0, \frac{\pi}{3}\right]$, by the Lagrange mean value theorem, it follows that $f$ is a contractive map.

By the fixed-point theorem, the recurrence sequence $\alpha_{k}=f^{k}(\alpha)$ converges to the unique fixed-point of $f$, i.e. $x=f(x)$, which implies $x=0$.

In Figure 4 a 'picture proof' illustrates the iterative process where $f^{k}\left(\alpha_{0}\right)$ converges to 0 for $x$-axis representing min angle $\alpha$.

Since $\lim _{k \rightarrow \infty} \alpha_{k}=0$, then also $\lim _{k \rightarrow \infty} \beta_{k}=\lim _{k \rightarrow \infty} \alpha_{k-1}=0$. Thus, $\lim _{k \rightarrow \infty} \gamma_{k}=\pi$ which proves the theorem.

Theorem 3. The family of triangulations generated by the conforming LE n-section from some initial triangulation of $\bar{\Omega}$ with $n \geq 4$ does not satisfy the maximum angle condition (2).

Proof: To prove the theorem, we show how to select an infinite sequence of subtriangles, among those generated by the conforming LE $n$-section, so that their maximum angles tend to $\pi$. First, we notice that the initial triangulation has at least one triangle, let us denote it by $T$. Now, we use the construction of "bad" triangles presented in the proof of Theorem 2 using $T$. Namely, we take as the sequence needed to prove our theorem the sequence $\left\{t_{k}\right\}$ constructed in the proof of Theorem 2. As their maximum angles tend to $\pi$ we obviously get what we want.

Now, we show that all these "bad" triangles $t_{k}$ are really contained among the set of triangles generated by the conforming LE $n$-section applied to $T$. First of all, the triangle $T$ can have several edges of the same length. When we apply the conforming LE $n$-section to $T$ in this case, we select some edge (randomly or according to some criterion) at the 
first step. We use the same edge to start the process of the classical $n$-section for $T$ as in Theorem 2. So, the triangle $t_{1}$ is really produced by the conforming LE $n$-section as the both versions of LE $n$-section coincide at the first step within $T$. Now we use the mathematical induction. Assume now that $t_{n}$ is contained among triangles produced by the conforming LE $n$-section. As it is clear that all $t_{k}$ for $k \geq 1$ are obtuse (i.e. there is always only one longest edge), the division stensil for both refinement versions within $t_{n}$ is the same. What remains is to notice that even though the conforming LE $n$-section "works" differently that the classical LE $n$-section, due to Theorem 1 , the conforming $n$-section sooner or later will refine $t_{n}$ and, therefore, $t_{n+1}$ will be among the subtriangles produced by the conforming LE $n$-section.

Acknowledgement: The first author was supported by Grant MTM2011-24766 of the MICINN, Spain.

\section{References}

[1] I. Babuška, A. K. Aziz. On the angle condition in the finite element method. SIAM J. Numer. Anal. 13 (1976), 214-226.

[2] R. E. Barnhill, J. A. Gregory. Sard kernel theorems on triangular domains with applications to finite element error bounds. Numer. Math. 25 (1976), 215-229.

[3] P. G. Ciarlet. The Finite Element Method for Elliptic Problems. North-Holland, Amsterdam, 1978.

[4] P. Jamet. Estimation de l'erreur pour des éléments finis droits presque dégénérés. RAIRO Anal. Numér. 10 (1976), 43-60.

[5] S. Korotov, M. Křížek, A. Kropáč. Strong regularity of a family of face-to-face partitions generated by the longest-edge bisection algorithm. Comput. Math. Math. Phys. 48 (2008), 1687-1698.

[6] M. Křížek. On semiregular families of triangulations and linear interpolation. Appl. Math. 36 (1991), 223-232.

[7] Á. Plaza, S. Falcón, J. P. Suárez. On the non-degeneracy property of the longest-edge trisection of triangles. Appl. Math. Comput. 216 (2010), 862-869.

[8] J. Suárez, T. Moreno, P. Abad, Á. Plaza. Properties of the longest-edge $n$-section refinement scheme for triangular meshes. Appl. Math. Letters 25 (2012), 2037-2039.

[9] A. Ženíšek. The convergence of the finite element method for boundary value problems of a system of elliptic equations (in Czech). Apl. Mat. 14 (1969), 355-377.

[10] M. Zlámal. On the finite element method. Numer. Math. 12 (1968), 394-409. 